\title{
Persée
}

\author{
http://www.persee.fr
}

\section{L'entreprise dans l'économie des conventions}

\author{
Pascal Ughetto \\ Revue économique, Année 2000, Volume 51, Numéro 1 \\ p. 51 - 73
}

Voir l'article en ligne

\section{Avertissement}

L'éditeur du site «PERSEE » - le Ministère de la jeunesse, de l'éducation nationale et de la recherche, Direction de l'enseignement supérieur, Sous-direction des bibliothèques et de la documentation - détient la propriété intellectuelle et les droits d'exploitation. A ce titre il est titulaire des droits d'auteur et du droit sui generis du producteur de bases de données sur ce site conformément à la loi n`98-536 du 1 er juillet 1998 relative aux bases de données.

Les oeuvres reproduites sur le site «PERSEE » sont protégées par les dispositions générales du Code de la propriété intellectuelle.

Droits et devoirs des utilisateurs

Pour un usage strictement privé, la simple reproduction du contenu de ce site est libre.

Pour un usage scientifique ou pédagogique, à des fins de recherches, d'enseignement ou de communication excluant toute exploitation commerciale, la reproduction et la communication au public du contenu de ce site sont autorisées, sous réserve que celles-ci servent d'illustration, ne soient pas substantielles et ne soient pas expressément limitées (plans ou photographies). La mention Le Ministère de la jeunesse, de l'éducation nationale et de la recherche, Direction de l'enseignement supérieur, Sous-direction des bibliothèques et de la documentation sur chaque reproduction tirée du site est obligatoire ainsi que le nom de la revue et- lorsqu'ils sont indiqués - le nom de l'auteur et la référence du document reproduit.

Toute autre reproduction ou communication au public, intégrale ou substantielle du contenu de ce site, par quelque procédé que ce soit, de l'éditeur original de l'oeuvre, de l'auteur et de ses ayants droit.

La reproduction et l'exploitation des photographies et des plans, y compris à des fins commerciales, doivent être autorisés par l'éditeur du site, Le Ministère de la jeunesse, de l'éducation nationale et de la recherche, Direction de l'enseignement supérieur, Sous-direction des bibliothèques et de la documentation (voir http://www.sup.adc.education.fr/bib/ ). La source et les crédits devront toujours être mentionnés. 


\title{
L'entreprise dans l'économie des conventions
}

\author{
Pascal Ughetto*
}

\begin{abstract}
L'économie des conventions développe une conception de l'entreprise mettant en valeur l'importance d'une instrumentation permettant au collectif de l'entreprise d'assurer le succès de ses produits à la vente. Ce succès est effectivement dépendant de la qualité (i.e. des caractéristiques) des produits, qui ne se présentent pas comme des biens anonymes mais comme des marchandises qui véhiculent avec elles la qualité des coordinations nouées autour de la production et du développement du savoir collectif. Cet apport renouvelle la représentation de l'entreprise mais aussi de l'articulation entre le micro et le macroéconomique, sans toutefois être à l'abri de certaines limites.
\end{abstract}

\section{ENTERPRISE IN THE “ECONOMICS OF CONVENTIONS”}

The economics of conventions develops a conception of enterprise which stresses the role of tools enabling the enterprise's community to ensure market success for its products. This success depends on the quality (i.e. the characteristics) of the products, which do not appear as impersonal goods but as commodities which carry the quality of the coordinations established around the production and the development of the collective knowledge. This contribution renews the representation of enterprise but also of the linkages between the micro- and the macro-economic levels, although it can exhibit some limits.

Classification JEL : D23, J41, L15, L16, L22

À première vue, l'économie des conventions ne se spécifie pas par un effort explicite et évident de construction d'une théorie de la firme. L'entreprise n'est pourtant pas absente des analyses de ce courant. Certains sociologues estiment même pouvoir déceler dans le modèle des économies de la grandeur de L. Boltanski et L. Thévenot [1991] des applications intéressantes à son propos, en dépit du fait, là encore, qu'on n'y trouve pas d'effort constitué d'élaboration d'une sociologie des organisations (H. Amblard et al. [1996]). La recherche en gestion, également, commence à s'ouvrir à la problématique conventionnaliste ${ }^{1}$. A leur tour, les économistes trouveraient certainement un intérêt à prendre acte de l'émergence d'une ligne d'analyse fournissant une compréhension spécifique des enjeux liés aux entreprises. À cet égard, c'est sans doute un exercice utile que de faire ressortir explicitement et de rassembler les éléments d'analyse de

* Université Paris-IX-Dauphine, IRIS, place du Maréchal-de-Lattre-de-Tassigny, 75775 Paris cedex 16.

1. Cf. Revue française de gestion, 112, janvier-février 1997. 
l'entreprise présents dans les écrits en termes de conventions et de tenter de dégager leur originalité par rapport aux approches existantes.

En économie, l'entreprise apparaît au sein de trois champs de problématiques, dont deux au moins sont destinés à dépasser le manque d'épaisseur de la fonction de production. Un premier champ est constitué par l'organisation comme forme de coordination alternative au marché (Coase, Williamson) et la représentation des relations internes à l'entreprise, soit en termes de hiérarchie (ibid.), soit en termes de relation d'agence au sein d'une firme vue comme nœud de contrats (Alchian et Demsetz). Le second champ est celui des modalités et procédures de la prise de décision au sein d'une organisation regroupant des individus aux intérêts divers et en conflit (Simon, théorie béhavioriste de la firme). Ces deux premières perspectives constituent les théories de la firme à proprement parler. Mais on aborde aussi les phénomènes d'entreprise lorsqu'on traite de la mise en œuvre du travail dans un cadre contractuel incomplet générateur d'incertitude sur la productivité. Ce sont les problématiques de la relation de travail ${ }^{1}$.

L'approche conventionnaliste, lorsqu'elle traite de l'entreprise, s'attache à développer conjointement ces trois dimensions ${ }^{2}$, réunissant ainsi des axes de recherche généralement séparés. Par exemple, l'analyse de la relation de travail fait directement partie du point de vue conventionnaliste sur l'entreprise. Il n'est pas indifférent que les auteurs du courant choisissent de procéder à un tel rassemblement de questionnements généralement dissociés par l'économie standard. C'est là le signe d'une approche repérant, dans les phénomènes d'entreprises, des enjeux différents vis-à-vis du fonctionnement économique : les questions économiques suscitées par l'entreprise ne se réduisent pas à celles, par exemple, des arrangements contractuels tels que décrits habituellement par les théories de la firme, et cela importe pour la compréhension de phénomènes économiques plus généraux (conception de la compétitivité, etc.). Nous verrons que l'originalité de l'approche conventionnaliste peut être située dans la volonté de se sortir de la question des arrangements inter-individuels pour se centrer sur la capacité d'un collectif à réussir, par la qualité de l'instrumentation de sa coordination, le passage des épreuves de vente de ses produits.

Avant de pouvoir donner sens à cet énoncé, il faudra commencer par isoler et porter au jour les éléments d'analyse conventionnaliste traitant de l'entreprise. Une première partie retiendra certaines analyses pour montrer en quoi, dans une optique en termes de conventions, l'entreprise est un collectif dont la coordination requiert une instrumentation des interactions entre ses membres. Une

1. Les développements contractualistes sur la relation de travail (théories des contrats implicites, du salaire d'efficience, etc.) approfondissent l'idée d'un découplage entre le moment où est signé le contrat de travail et la mise en œuvre du travail par la suite : en en tirant l'idée d'un risque de comportement opportuniste de l'une ou de l'autre des parties, ils s'intéressent aux contrats venant réduire l'incertitude sur le comportement de l'autre dans le déploiement de la relation. En général, la « théorie standard étendue » (cf. infra) dissocie ces développements des théories de la firme.

2. La seconde, il est vrai, a été moins développée : elle reste plutôt à l'état latent sous la forme des registres de justification opposant, à l'intérieur des entreprises, des arguments relevant de «natures » différentes, notamment « industrielle » et « marchande » (cf. L. Thévenot [1989]). 
seconde partie, se concentrant sur d'autres analyses, révélera l'enjeu de la qualité des coordinations au sein du collectif : l'enjeu est de réussir le passage des épreuves de réalisation de la vente des produits, qui mettent précisément à l'épreuve ces coordinations. Sur cette base, il sera possible, dans une troisième partie, de s'interroger sur l'apport de l'économie des conventions sur la question de l'entreprise et de le discuter.

\section{L'ENTREPRISE : UN COLLECTIF DONT LA COORDINATION NÉCESSITE UNE INSTRUMENTATION}

Les auteurs se reconnaissant dans la problématique en termes de conventions ont développé l'idée, à partir d'une discussion de la théorie "standard », qu'il était impossible de concevoir des relations d'échange qui ne soient pas instrumentées par des objets échappant à la dimension contractuelle ou à l'hyperrationalité. Les règles en font partie. L'entreprise constitue, de ce point de vue, un support privilégié de démonstration puisque, « s'il est un lieu où l'on suit des règles, c'est bien celui-là » (O. Favereau [1995], p. 515). Une autre notion avancée par les analyses conventionnalistes est celle de «formes ». Au-delà du vocabulaire, l'idée commune est celle du caractère réducteur de la représentation de l'entreprise comme fonction de production aussi bien que comme lieu de contrats inter-individuels internes : en lieu et place de cela, il faut s'interroger sur une certaine alchimie des interactions au sein du collectif de l'entreprise, qui ne parviennent à produire et à contribuer au développement de l'entreprise que grâce à l'instrumentation sur lesquelles elles s'appuient. La problématique des investissements de forme et la réflexion sur les règles salariales creuse ce problème, tandis que l'approche en termes de règles d'O. Favereau le replace au sein d'un questionnement beaucoup plus général sur la représentation pertinente des économies.

\section{Les investissements de forme et les règles comme instruments d'évaluation}

Le modèle des investissements de forme (L. Thévenot [1985]), élaboré par F. Eymard-Duvernay et L. Thévenot, peut s'appliquer à un domaine assez large, susceptible a priori de dépasser l'entreprise. Les opérations de mise en forme d'une relation sont, en effet, définies d'une manière très étendue, comme étant celles qui « consistent à régler une relation, à transformer une interaction soumise à l'incertitude, à la négociation, en un échange automatique où les propriétés des individus qui sont mis en relation n'interviennent plus » (F. EymardDuvernay [1986], p. 239 - italiques d'origine). L'opération d'investissement étant définie " comme l'établissement, coûteux, d'une relation stable, pour une certaine durée » (L. Thévenot [1985], p. 27 - ibid.), elle peut s'appliquer, audelà de l'investisseur traditionnel qu'est l'entreprise, à « toute personne engagée, pour un certain temps, par le sacrifice d'une satisfaction immédiate de ses désirs » (ibid.). Par ailleurs, l'objet de l'affectation coûteuse de ressources, les formes, est également susceptible de recevoir une définition très large. Une forme est l'objet qui, en jouant un rôle d'instrumentation, rend possible la 
stabilisation d'une relation. Il existe ainsi des formes d'État (comme le diplôme, qui institue une équivalence entre des individus a priori divers, de telle sorte que la négociation pour l'établissement d'une relation va pouvoir dépasser les caractéristiques particulières des personnes et l'incertitude qui leur est liée pour s'appuyer sur le fondement plus stable que constitue l'objectivation offerte par la «forme titrée »). Il existe également des formes intermédiaires (de branche, par exemple) et des formes spécifiques, établies par un acteur particulier dans le cadre des interactions qui le concernent en propre.

Or, un lieu spécialement riche en formes spécifiques est l'entreprise (notamment celles régissant l'usage de la main-d'œuvre). C'est en fait la représentation, traditionnelle pour l'économiste, de la fonction de production qui est mise en cause : la production ne nécessite pas uniquement des équipements, des matières premières et des travailleurs, mais, de façon aussi cruciale, tout un ensemble d'objets aussi divers que des règlements, des normes, des consignes, des procédures de standardisation, etc. L'exemple est donné des préceptes et « instruments » de F.W. Taylor (chronomètre, tâche, instructions écrites...), qui apparaissent comme des « investissements » permettant de « réaliser matériellement les conditions d'une fonction de production conforme aux formulations de la théorie économique, avec un facteur travail assimilable à une quantité de temps, consommable sans aucun effet d'immobilisation de main-d'œuvre " (L. Thévenot [1985], p. 32). Bref, au-delà de l'illustration, il ressort que l'entreprise ne peut être pensée comme une simple fonction de production, mais qu'elle se présente comme un lieu de coordination d'interventions qui ne peuvent s'établir sans une instrumentation coûteuse.

La conception des règles peut mettre l'accent sur les conditions de l'accord entre les acteurs de l'entreprise sous un angle moins unilatéral que dans le modèle des investissements de forme, qui se centre fortement sur les formes instituées par l'employeur. La démonstration s'offre alors comme support la discussion sur la productivité et la qualité du travail. Elle peut s'énoncer principalement en termes de conventions (et secondairement en termes de règles, qui apparaissent, dans ce cas, comme des instrumentations des conventions) : c'est le cas des analyses de R. Salais dont il sera rendu compte dans la seconde partie. Mais elle peut aussi se centrer sur les règles (et seulement secondairement sur les conventions, qui constituent alors un arrière-fond) : ce sont les travaux de B. Reynaud [1992; 1994] sur les règles salariales.

Il s'agit alors de reprendre le thème de l'incomplétude du contrat de travail. En fait, l'analyse standard du contrat de travail (B. Reynaud [1988]) révèle que tous les théoriciens néo-classiques ne réduisent pas le travail à une marchandise comme les autres, si l'on entend par là qu'ils reconnaissent l'existence d'une dissociation entre l'achat d'unités de travail et la réalisation même de ce travail au cours de l'acte de production. De même, ils en déduisent correctement le caractère incomplet du contrat de travail, c'est-à-dire le fait que, partant de l'incapacité qui le caractérise à décrire tous les états de la nature à venir et les comportements requis correspondants, ce contrat ne peut se suffire à lui-même et nécessite d'être complété. Certains perçoivent bien, en outre, que l'incertitude pesant sur la qualité du travail ne peut être résolue par le système des prix et impose le recours à une coordination par les règles et notamment les règles salariales. Mais l'illusion commune de tous ces théoriciens est de croire à la possibilité de solutions qui permettent de résoudre définitivement le problème. 
En fait, c'est, selon B. Reynaud, la spécificité de l'hétérodoxie que de considérer comme irréductible l'incomplétude du contrat de travail : les règles salariales ne peuvent jamais la supprimer intégralement ; elles contribuent à son exécution la plus conforme aux attentes sans être en mesure d'en garantir un résultat parfait.

Il conviendrait donc de voir la règle salariale, moins comme une solution définitive, que comme un instrument d'évaluation rendu nécessaire par la relation et servant à la discussion des contractants autour de la réalisation des attentes en matière de qualité du travail. Ce qui autorise la règle à jouer ce rôle, c'est la double dimension qu'elle présente : étalon, elle évalue les actions passées ; modèle, elle définit les comportements à venir. La règle-étalon, en se diffusant, véhicule avec elle un modèle de référence, c'est-à-dire une représentation que les individus acceptent et partagent - ce qui permet d'éviter des discussions incessantes - et qui désigne un objectif à atteindre (ibid., p. 57-58). B. Reynaud fait valoir que « [1]'adoption d'une règle salariale, comme sa diffusion, repose en très grande partie sur la légitimité de la règle » (ibid., p. 80 italiques d'origine). Toutefois, en dépit du fait que la règle instrumente une coordination interne à l'entreprise entre deux catégories d'acteurs asymétriques - l'employeur et les travailleurs -, le processus de diffusion semble considéré par l'auteur principalement du seul point de vue de l'employeur. L'importance de l'asymétrie paraît plus directement décelée par R. Salais ([1989], p. 216-217) qui concède que, par les règles, « l'inégalité des personnes est rendue apparente dans leur inégale capacité à imposer leurs principes d'équivalence dans la négociation ». Les règles, effectivement, tirent, selon cet auteur, leur légitimité de leur double fonction : tout d'abord, elles hiérarchisent les principes d'équivalence entre travail et salaire, d'une part, et entre travail et produit, d'autre part ( $c f$. infra), en subordonnant le premier - représentant le point de vue du salarié - au second - correspondant au point de vue de l'employeur; par ailleurs, elles sont utilisées dans la résolution des désaccords au cours de la relation.

\section{Les dispositifs cognitifs collectifs}

Le lien entre l'entreprise et les règles peut également être analysé à partir de l'engagement d'un programme de recherche « cognitiviste » en économie, avec en perspective l'objectif ambitieux de contribuer à la production d'une représentation pertinente de l'architecture générale d'une économie. Cette perspective, ouverte par $\mathrm{O}$. Favereau, consiste à renouer avec le «projet radical » de Keynes ${ }^{1}$, en considérant que la particularité de ce dernier tient dans l'accent mis sur les dispositifs variés de traitement de l'incertain : "procédures réglementaires ou conventionnelles sur les marchés financiers, procédures organisationnelles sur les marchés des biens et de facteurs » (O. Favereau [1989a], p. 321 ; voir également O. Favereau [1986]). Cette perspective rencontre le mouvement qui, ces vingt dernières années, a conduit la «théorie standard » (celle dont les deux piliers sont le marché et la rationalité substantive) à découvrir l' « organi-

1. O. Favereau [1988] distingue, chez Keynes, un « projet pragmatique » et un « projet radical ", tous deux destinés à rendre compte de la possibilité d'un chômage involontaire massif, mais le premier sur la base d'une rupture minimale avec le modèle néo-classique, le second à partir de remises en cause plus affirmées. 
sation », c'est-à-dire les phénomènes de coordination par les règles en opposition au marché. Ce faisant, la théorie standard (TS) s'est transformée en " théorie standard étendue » (TSE). L'opération a consisté, pour elle, à rendre compte de l'organisation au moyen d'un développement du pôle de la rationalité et d'une recomposition du pôle du marché. L'homogénéisation des représentations a été faite grâce au contrat, qui a servi de réalité unificatrice $(O$. Favereau [1989a, b]). Mais, dans cette opération, ce qui s'est trouvé gagné en étendue a été perdu en cohérence : l'organisation a été additionnée au marché, mais avec des résultats problématiques. D'une part, il devient impossible d'avoir recours à la simplification walrassienne qui permettait de définir l'économie comme un ensemble de marchés interdépendants, cela sans que soit proposée une autre définition cohérente (« Nous ne savons plus ce qu'est une économie »); par ailleurs, des marchés auxquels se trouvent adjointes des organisations ne peuvent plus être dépeints sur le modèle du marché boursier («Nous ne savons toujours pas ce qu'est un marché ») ; enfin, le tout est proposé sans définition acceptable des règles ( «Nous ne savons pas encore ce qu'est une règle ») (O. Favereau [1989a]). La résolution de ces trois problèmes est entreprise sur la base de l'élaboration d'une théorie des règles et d'une définition de l'organisation comme marché interne du travail.

Le recours aux autres sciences sociales permet d'élaborer une théorie des règles comme dispositifs cognitifs collectifs. L'approche cognitive vise à dépasser les limites rencontrées par la conception standard des règles comme « règles-contraintes » (dont l'exemple type est la contrainte budgétaire du consommateur) et comme « règles-contrats » (adoptées par consentement mutuel). Dans ce dernier cas, qui modélise des arrangements contractuels entre individus, directs ou indirects (salaire d'efficience, « convention » à la Lewis...), la nécessité apparaît toujours d'un tiers ou d'un objet extérieur (réputation...) qui entre en contradiction avec la volonté de ne rendre compte que de strictes intentionnalités. Une voie plus nuancée se dessinant entre ces deux extrêmes consiste à considérer les règles du point de vue des économies de savoir qu'elles autorisent (O. Favereau [1989a], p. 292 et suiv. ; [1989b], p. 82 et suiv.). Reprenant comme illustration l'exemple traditionnel, dans les disciplines cognitives, de la conduite d'une automobile (qui ne nécessite pas de connaissance de la mécanique interne de celle-ci), $O$. Favereau fait apparaître les règles comme des dispositifs cognitifs qui cristallisent un savoir permettant de réaliser une action sur la seule base de la connaissance du mode d'utilisation et de fonctionnement de la technique - qu'il n'y a qu'à suivre comme règle - sans que soient requises des connaissances théoriques plus approfondies. Dit autrement, la règle permet de se limiter à la question du " comment » (faut-il faire ?) sans se préoccuper du «pourquoi » (faut-il le faire ?). Ces dispositifs cognitifs ont une dimension collective : la possibilité pour chacun d'être partiellement ignorant repose sur l'appui qu'il peut trouver dans le savoir des autres. Les règles concrétisent un savoir collectif. C'est dans cette perspective que prend son sens le contrat de travail, dont l'incomplétude n'est pas le problème mais « la solution ») (O. Favereau [1989a] p. 295). Une liste exhaustive des situations auxquelles aura à se confronter le collectif de travail n'étant pas envisageable, le contrat de travail va non seulement - comme l'avait vu H. Simon [1951] introduire de la flexibilité en rendant modulables les affectations, mais surtout résoudre la tension de l'incertitude au moyen de l'articulation des apprentissages individuels et collectifs : au lieu de définir des affectations et des comportements 
pour toute occurrence possible (ce qui est irréaliste), le contrat de travail insère en fait les salariés dans un savoir collectif qui va définir des routines d'action mais en laissant ouverte la possibilité d'une adaptation, par apprentissage, de ces routines. L'entreprise est ainsi bien autre chose qu'un lieu de relation d'autorité, c'est un lieu d'apprentissage, et la flexibilité n'est pas tant celle des affectations révisables que celle du développement et des déplacements de savoirs individuels et collectif.

Il ne reste alors qu'à adjoindre l'apport de P. Doeringer et M. Piore sur les marchés internes du travail, en vertu duquel on doit concevoir les règles comme s'opposant fondamentalement à la mise en concurrence des travailleurs avec les offreurs de travail extérieurs. À partir de là, il devient possible de dresser une typologie des marchés, sur la base de laquelle pourra être proposée une nouvelle représentation de ce qu'est une économie, articulant marché et organisation de façon cohérente. O. Favereau ([1989a], p. 304 et suiv.) suggère une distinction entre les organisations de marché (i.e. les marchés d'actifs financiers, en tant qu'ils doivent être soumis à une règle stricte organisant la confrontation offredemande), les marchés d'organisation (les marchés de produits, en ce qu'ils se présentent avant tout comme des espaces de concurrence entre organisations) et les organisations anti-marché (les marchés internes soustrayant les travailleurs aux mécanismes du marché externe). Se trouve ainsi assumée la rupture avec le projet walrassien d'extension du modèle du marché boursier à tous les marchés. Les entreprises sont des organisations soumises à une contrainte de rentabilité et la rentabilité pose le problème de la viabilité de leur marché interne. Le prix qu'elles parviennent à obtenir pour leurs produits (étant donné les caractéristiques, la qualité, de ceux-ci) constitue, sous un autre angle de vue, une source de financement de leur marché interne. Le problème alors n'est pas, pour elles, celui d'une recherche de l'adéquation à la demande par un simple ajustement des prix proposés mais un jeu sur le rapport entre les caractéristiques des biens présentés - caractéristiques autorisées par l'état de leur savoir collectif - et les prix acceptés sur le marché pour ces caractéristiques. Il s'agit que les entreprises, par «modifications successives de leur Savoir Collectif, trouvent leur niche dans l'espace des qualités » (ibid., p. 312). Dès lors, le renversement par rapport à la théorie néo-classique est complet. Très loin de juger - négativement l'organisation et la règle à la mesure des entraves qu'elles occasionnent vis-à-vis de mécanismes de marchés posés comme fondamentaux, ce sont les organisations qui sont placées comme premières, de sorte que le problème posén'est pas celui de la restauration de mécanismes de marché compris comme ceux des marchés financiers, mais celui de l'apprentissage des firmes : «... l'efficience des procédures de type [organisations anti-marché] ne doit pas être jugée en statique à l'aune des imperfections qu'elles entraînent dans un système walrasien du type [organisations de marché]. Cette efficience est dynamique : elle se mesure en termes d'Apprentissage Organisationnel, rendu possible par le transfert du jeu de la concurrence, du marché du travail vers le marché des biens. » (Ibid.) Une économie doit donc être conçue comme une population d'organisations se disputant la ressource rare qu'est le pouvoir d'achat global et structurée par une double contrainte : circulatoire au niveau des marchés de produits (le circuit keynésien) et walrassienne au niveau des marchés d'actifs financiers. Les entreprises sont effectivement soumises aux deux appréciations, des 
consommateurs et du système financier. Une relecture du modèle IS-LM ${ }^{1}$ est alors proposée qui tente d'articuler circuit (dont rendrait compte la courbe IS) et marché (LM) et qui permettrait de rejoindre et d'offrir un fondement théorique à l'explication keynésienne du chômage massif en vertu de laquelle « le processus de coordination par le marché contraint (à travers le choix d'un horizon trop court) le processus de coordination par le circuit à ne rendre possible la reproduction des organisations, qu'avec un niveau de Demande Effective trop faible pour employer tous les actifs » (ibid., p. 321). La pensée de l'organisation et des règles débouche alors sur une théorie du chômage de masse : " Au total, les sociétés hétéronomes connaîtraient leurs crises les plus graves lorsque les principes d'organisation ne sont plus à la hauteur des principes de marché. L'interdépendance généralisée entrave alors les dynamiques d'accumulation du Savoir Collectif par les organisations » (ibid. - italiques d'origine).

L'approche proposée par O. Favereau contribue donc, avec les précédentes, à imposer la reconnaissance d'un premier impératif dans l'appréhension des entreprises : une entreprise est un lieu d'instrumentation des interactions entre membres du collectif qu'elle représente. Au fond, il ne suffirait pas d'agencer des facteurs productifs au sein d'une fonction de production ni même d'assurer la coordination interne par des arrangements contractuels : tout cela ne déboucherait sur aucune production si cela n'était étayé par un apprentissage (ou développement du savoir collectif incarné dans les règles) et un investissement dans les règles de stabilisation et de gestion des relations entre acteurs économiques. Mais l'approche d'O. Favereau va au-delà : comme les analyses qui vont maintenant être présentées - qui ont pour avantage d'expliciter précisément ce point -, elle suggère l'enjeu qui est attaché aux modalités de la coordination au sein du collectif : il s'agit de la confrontation de l'entreprise au marché, dans une conception où le succès de cette confrontation reposera fondamentalement sur la qualité des coordinations internes.

\section{LES CONVENTIONS EN VUE DE LA FABRICATION ET DE LA VENTE DES «PRODUITS »}

Les analyses de F. Eymard-Duvernay et de R. Salais donnent une importance particulière aux " produits» des entreprises. Le produit, terme apparemment banal, mais qui sous-entend une position opposée à celle de l'analyse standard : loin d'être homogènes, les biens présentés sur un marché véhiculent avec eux les caractéristiques des entreprises qui les ont produits ; la «qualité » du produit (i.e. ses caractéristiques) révèle notamment la qualité du travail déployé dans l'entreprise qui en est à l'origine, ce qui renvoie à la qualité des coordinations internes. Le produit assure l'interface entre l'organisation et le marché. Sur cette base, les auteurs en question font ressortir la pluralité des modes de coordination possibles et suggèrent ainsi des questionnements originaux renouvelant l'analyse de la période actuelle et de ses enjeux.

1. Voir également O. Favereau, L. Thévenot [1996]. 


\section{Les conventions de qualité et les modèles d'entreprises}

La qualité des produits est, aux yeux de F. Eymard-Duvernay, un terrain privilégié pour dénoncer l'illusion qu'il y a à vouloir opposer le marché (auquel l'efficacité serait entièrement associée) et la règle (qui constituerait une protection artificielle).

La théorie standard considère des biens dont la qualité est parfaitement objective, les prix n'ayant alors qu'à s'ajuster en fonction des raretés relatives. Un certain nombre d'auteurs font apparaître que, en présence d'asymétries d'information entre l'acheteur et le vendeur, le prix perd cependant ses propriétés de réalisation de l'équilibre du marché et se trouve saisi comme objet stratégique par les rationalités des agents (« sélection contraire ») : les mécanismes du marché se trouvent fortement perturbés, ce qui justifie l'existence de règles. En dépit de leur apport, ces travaux ont, néanmoins, pour limite de ne pas poser la question de la définition de la qualité : «L'incertitude, source des fonctionnements perturbateurs, est vue comme le résultat d'un défaut d'information. Les qualités "réelles" des biens sont définies sans ambiguïté, de façon exogène, comme inscrites dans les biens. » (F. Eymard-Duvernay [1989], p. 332.) Cette hypothèse peut être jugée rapide : « De nombreuses observations conduisent à l'idée que les produits n'ont pas toujours le degré d'objectivation qu'on leur suppose. La transaction peut porter sur un "service", être faite de relations personnelles, non médiatisées par des objets, des règles. » (F. Eymard-Duvernay [1986], p. 240.) Dans ces situations, la qualité ne s'impose pas comme un fait objectif : il faut, au contraire, supposer qu'elle fait l'objet d'une construction. Selon une logique issue de la problématique des investissements de forme, on pourra alors considérer que des « outils » sont requis pour régulariser la relation entre le vendeur et l'acheteur : la répétition, qui autorise la production d'habitudes, peut être utile, voire la marque, qui a pour propriété d'étendre l'espace de validité du produit, ou encore la normalisation - ce que F. Eymard-Duvernay nomme la "qualification » du produit. Au-delà, il y a possibilité d'orienter la réflexion vers les conventions de qualité susceptibles d'être construites (F. Eymard-Duvernay [1989]). Celles-ci font correspondre à un type de produit la mobilisation, dans la production, de ressources qui sont adéquates et cohérentes en ce qu'elles concourent efficacement à l'obtention du type de qualité choisi. Ainsi, les produits « industriels » se verront associés par la convention de qualité correspondante une affectation des ressources dirigée à titre principal vers des équipements matériels, et la recherche de l'efficacité passera par la spécialisation des unités de production et l'allongement des séries. Une seconde convention de qualité associe les produits "de marque » et la mobilisation de ressources dans des investissements spécifiques et immatériels. Enfin, une entreprise qui se spécialiserait sur les produits "marchands " (c'est-à-dire très sensibles aux variations de la demande) devrait, pour être efficace, développer une convention de qualité qui s'attacherait à favoriser la variabilité des ressources elles-mêmes, du point de vue de leur coût. Les conventions de qualité pointent par conséquent le doigt sur des variables de gestion dont la pertinence diffère suivant les cas : si, par exemple, la constitution de stocks est justifiée par une convention de qualité industrielle (puisqu'elle est la condition ou la contrepartie du fonctionnement de processus productifs focalisés sur la recherche d'économies d'échelle), elle apparaîtra, dans l'optique d'une convention marchande, comme une irrationalité de gestion à combattre (puisque la variabilité de 
la demande exige que les ressources soient également soumises à une logique de variabilité). Selon ce schéma, la période actuelle apparaît marquée par la résurgence du marché et des « produits marchands » au détriment des produits « industriels ». Aucune garantie de stabilité ne semble cependant attachée à cette nouvelle configuration : « Si les produits purement industriels sont actuellement mis en difficulté, les activités immergées dans le marché, sans bénéficier des ressources industrielles, ne constituent pas non plus une assise solide pour le développement de l'économie. Soumis en permanence aux fluctuations du marché, les acteurs n'ont pas la capacité de donner consistance à des entreprises fondées sur des liens durables et l'accumulation de ressources industrielles. » (Ibid., p. 356.)

À partir du moment où l'on admet, comme il vient d'être fait, l'existence, pour les entreprises, d'une pluralité de modes de coordination possibles, l'entreprise n'est plus comprise sur la base du modèle unique constitué par une fonction de production qui exprimerait une rationalité identique pour toutes les firmes. Apparaissent, au contraire, plusieurs modèles d'entreprises (F. EymardDuvernay [1987]). Dans l'utilisation de leurs ressources à des fins de constitution du produit, les entreprises ne sont pas guidées par une logique uniforme : chacune se tourne plutôt vers l'une des logiques générales que le chercheur peut repérer. On observe ainsi une diversité des ressources engagées par les entreprises, au-delà des ressources classiquement prises en compte par l'économie : les réglementations, mais aussi les relations et l'histoire locales ou la tradition en font partie, cela « au même titre que les machines ou le travail car elles ont un coût, une efficacité dans l'acte de production et elles exigent un investissement particulier » (P. Boisard, M.-T. Letablier [1987]), p. 3). Sur cette base, on peut distinguer divers modèles d'entreprises, différenciés par les types d'usage des ressources : modèle « domestique » tirant parti des relations de proximité, de connaissance, d'ancrage local et traditionnel, pour produire, par exemple, des biens de tradition; modèle industriel, fondé sur le choix de consacrer des ressources à des investissements de forme en standardisation; etc. La mise en valeur des coordinations permettant de donner forme au produit et conditionnant sa «qualité » aurait ainsi tendance à s'étendre au réseau dans lequel opère l'entreprise ${ }^{1}$.

\section{Les conventions du travail et les mondes de production}

La démarche de $\mathrm{R}$. Salais, à la différence de la précédente, n'est pas tant de rendre compte du mode de constitution de la relation d'échange en dépit du

1. Par ailleurs, peut également être examinée l'incidence des coordinations sur le produit du point de vue de la conclusion de la transaction. De la même manière qu'elle explique que le produit véhicule avec lui la qualité des coordinations internes ayant présidé à sa fabrication, l'analyse conventionnaliste peut, en effet, développer ce qu'il en ressort du point de vue de la réputation du produit et de sa capacité à s'écouler sur le marché. Lorsqu'il existe une incertitude sur la qualité du produit, non pour des problèmes d'asymétrie d'information mais parce que les qualités du produit sont déterminées dans le cours même de l'échange (services...), l'organisation, à la différence de l'individu isolé, peut être perçue comme donnant une assurance sur la fiabilité de la prestation à venir (F. Eymard-Duvernay [1994]). 
manque d'objectivité du produit que de s'interroger sur les processus par lesquels se réalise le produit en dépit de l'incertitude qui pèse sur les divers moments concernés (conclusion du contrat de travail, mise en œuvre du travail, vente du produit sur le marché). La singularité de son analyse par rapport à l'analyse standard étendue est d'insérer la relation de travail dans une problématique du produit qui la dégage du simple champ théorique du marché du travail (à laquelle la restreignait la seule prise en compte des deux phases de négociation et d'application du contrat de travail) pour adopter un point de vue plus global (grâce au fait que l'analyse est étendue jusqu'à la prise en compte de la phase de « réalisation » de la vente du produit sur le marché). L'économie du travail et l'économie industrielle se trouvent ainsi directement intégrées : le travail participe pleinement à la compréhension des phénomènes d'entreprise et du fonctionnement économique global. Sont, dès lors, amenées à prendre une importance décisive les conventions du travail.

À la base, se situe un double constat : «Le principe de la relation de travail échappe au paradigme du marché parce que la relation se déroule dans le temps et parce que l'objet véritable de l'échange - le produit - n'existant pas encore, n'est pas présent au moment où l'échange doit se faire. » - «Mais, en même temps, la relation de travail se déroule dans un cadre général marchand; le produit verra sa valeur prévue validée ou non par son prix global lors de la vente des produits. » (R. Salais [1989], p. 199.) ${ }^{1}$. Deux conventions permettent aux acteurs économiques de dépasser ce dilemme. La première substitue au produit, qui n'existe pas encore, le salaire et établit donc une équivalence entre temps de travail futur et salaire: c'est la convention de productivité. La seconde, ou convention de chômage, établit une équivalence entre le temps de travail et le produit et considère la vente du produit (son « épreuve de réalisation ») comme une procédure d'évaluation de la convention de productivité. L'employeur est évidemment intéressé prioritairement par l'obtention de la seconde équivalence mais doit passer par la première pour l'obtenir. Un blocage devrait, au moment de la conclusion du contrat de travail, résulter du fait que rien ne lui garantit l'obtention future d'une équivalence temps-produit conforme à ses attentes (vente du produit et prix satisfaisant). Deux aléas pèsent, en effet, sur la relation, l'un externe, portant sur la demande, l'autre interne, tenant à la qualité du travail et à l'effort qui, en dépit d'une objectivation technologique et de règles de contrainte, restent sous la maîtrise des travailleurs. Seule l'existence de la convention de chômage lui permet de passer outre et de conclure le contrat, par l'assurance qu'elle lui offre de pouvoir répondre à une épreuve de réalisation défavorable par une réduction de l'activité (diminution de la durée travaillée, des effectifs...).

Une convention est définie comme " un système d'attentes réciproques sur les compétences et les comportements, conçus comme allant de soi et pour aller de soi » (ibid., p. 213). L'efficacité de la coordination requiert que les personnes se comprennent mutuellement, c'est-à-dire qu'elles puissent ajuster leurs attentes respectives grâce à l'utilisation d'un cadre de référence commun. Les

1. On retrouve là la base de la problématique marxienne distinguant le rapport d'exploitation et le rapport marchand et en déduisant que la plus-value est créée dans la circulation et en même temps en dehors de celle-ci. 
conventions du travail, quant à elles, se définissent par l'articulation qu'elles autorisent entre qualité du travail et qualité du produit. C'est en fait de la qualité du produit qu'il convient de partir ${ }^{1}$. Des grandes classes de produits peuvent être repérées en utilisant deux critères. D'une part, en se positionnant sur le marché et en adoptant le point de vue du demandeur, on peut distinguer les produits selon qu'ils sont «définis a priori par le producteur sans considération des individus singuliers auxquels ils pourraient s'adresser » (ibid., p. 35) - ce sont alors des produits génériques - ou qu'ils s'adressent à une demande considérée comme éminemment singulière - ce sont des produits dédiés. D'autre part, en se référant, cette fois, au critère de la technologie et du procès de production selon le point de vue du producteur, on distinguera les produits spécialisés, dont la propriété est d'incorporer l'usage d'actifs spécialisés (savoir-faire idiosyncrasique...), et les produits standardisés dans lesquels, au contraire, les personnes qui les fabriquent ne laissent aucune particularité personnelle. Ces derniers mobilisent essentiellement des économies d'échelle, alors qu'il s'agit d'économies de variété pour les produits spécialisés. Le croisement des deux catégorisations délimite des mondes possibles de production, c'est-à-dire des registres d'action et des cadres de référence au sein desquels peut être conçue la coordination entre les personnes. Ils sont au nombre de quatre. Le monde de production industriel est celui qui associe produit générique et produit standardisé. L'illustration typique est celle des biens fabriqués à la chaîne. Lorsque le produit est, non plus générique, mais dédié, il s'insère dans un monde de production marchand. Celui-ci est, beaucoup plus que le précédent, sensible aux exigences d'adaptation instantanée et permanente à la demande, par le prix et la qualité (réduction des délais de conception et de livraison...). Dans le cas de produits spécialisés, le monde de production sera dit interpersonnel pour un produit dédié et immatériel pour un produit générique. Ces deux dernières configurations correspondent à des situations où l'interaction entre le producteur et le demandeur doit être forte dans le but de définir et de construire conjointement le produit, avec la contrainte supplémentaire pour le monde de production immatériel que, au début de la relation, on ne conçoit même pas la forme que prendra le produit, ni même s'il pourra se concrétiser, ni quand cela se fera (ibid., p. 54).

Les conventions du travail se déduisent de la classification des mondes possibles (R. Salais, M. Storper [1993], p. 75 et suiv. ; R. Salais [1991 ; 1994]). À chaque monde possible correspond une convention de productivité à travers laquelle peut être envisagée une cohérence entre la qualité du travail et la qualité du produit (avec des modes de flexibilité différents, permettant de répondre à des aléas eux-mêmes distincts, des formes de salaire et des formes d'évaluation de la qualité également différents, etc.). De même, les conventions de chômage sont spécifiques à chacun des mondes possibles. Ces conventions viennent assurer une cohérence entre la qualité du produit définie selon l'un des quatre mondes possibles et la qualité du travail, travail sans lequel il n'y a pas de produit envisageable.

1. Précisons que, comme cela s'entendait également dans les travaux de F. EymardDuvernay, la notion de "produit » ne suggère ici aucune nécessité de matérialité physique (R. Salais, M. Storper [1993], p. 57-58). 
À mesure que, grâce notamment aux conventions du travail, le produit franchira avec succès les épreuves de réalisation, un monde possible se transformera en monde réel de production. Tout l'enjeu des mutations en cours serait alors, pour la spécialisation de l'économie française, d'opérer un déplacement du monde réel de production industriel, jusqu'alors dominant dans les produits des entreprises, vers les mondes possibles interpersonnel et immatériel (secondairement vers le monde marchand), qui sont désormais porteurs du meilleur potentiel du point de vue de la spécialisation et de la compétitivité d'une économie comme celle de la France. Il s'agit de construire, "autour de trajectoires de produits, des mondes réels, c'est-à-dire des structures de coordination, de production et d'échange douées d'une propriété d'autodéveloppement. La particularité de chacun de ces mondes réels est de créer de façon auto-entretenue des produits à partir d'agencements d'actions et de ressources s'éloignant du monde possible industriel et empruntant aux autres mondes possibles » (ibid., p. 120). La difficulté est cependant que les conventions existantes, y compris celles qui sont extérieures à l'entreprise (convention de l'État, exprimant les attentes quant à la présence de l'État dans le fonctionnement économique, conceptions patronales et syndicales de l'entreprise et de l'emploi...) exercent une force de rappel qui ramène en permanence vers le monde de production industriel et ses produits.

Le thème des conventions du travail et des conventions de qualité fait apparaître clairement l'importance des dimensions fondant la qualité des produits des firmes. Entreprise et marché ne sont pas deux univers indépendants. Le produit assure la continuité entre les deux puisque son succès repose sur l'appréciation de sa qualité (de ses caractéristiques), qui a elle-même pris naissance dans les conventions internes à l'entreprise, conventions du travail et de la qualité.

\section{LA « NATURE DE LA FIRME »CONVENTIONNALISTE}

Le passage en revue auquel il vient d'être procédé suggère que plusieurs angles de vue sont susceptibles d'être retenus pour appréhender l'entreprise dans une perspective conventionnaliste. Cela n'enlève rien à la possibilité de cerner une «nature » précise de la firme conventionnaliste.

\section{Fait productif, confrontation collective à l'incertitude et instrumentation de la coordination}

Les axes de recherche recensés convergent vers une vision relativement cohérente de l'entreprise : celle-ci apparaît comme un lieu de confrontation collective à l'incertitude dans une perspective de rentabilité. Un premier niveau de définition, effectivement, est l'énoncé de l'objectif ou de la contrainte de rentabilité qui caractérise l'entreprise. Pour O. Favereau ([1989a], p. 276), « on pourrait réserver le terme "Entreprise" aux organisations dont la contrainte de cohérence collective implique un résultat non seulement objectivable mais objectivable en termes de profit monétaire ». À partir de là, l'entreprise peut être définie très explicitement. Soit à la manière d'O. Favereau ([1989b], p. 90) : elle correspond, dans ce cas, à des « dispositifs cognitifs collectifs aménageant, au 
moyen de contrats et de contraintes, des interactions individuelles de nature à desserrer - par un processus d'apprentissage collectif - une contrainte globale de rentabilité minimale ». Soit à la manière de R. Salais et M. Storper ([1993], p. 23) : «L'entreprise est une organisation collective destinée à affronter et surmonter ces épreuves de réalité que sont la production et la vente des produits dans des conditions satisfaisantes de profitabilité. »Dans les deux cas, ressort clairement l'idée que la réalisation du produit et la rentabilité de l'entreprise sont à la fois le motif expliquant la réunion des personnes dans un collectif et l'épreuve à l'issue incertaine dont la réussite repose fondamentalement sur les capacités qu'offrent cette réunion en collectif et l'exploitation plus ou moins efficace qui en est faite. Dit autrement, l'économie des conventions met en lumière le fait que l'étude de l'entreprise et de ses réussites (ou de ses échecs) doit intégrer de façon prioritaire l'analyse de la qualité des coordinations et de leur efficacité du point de vue de la fabrication du produit et de sa vente, étant entendu que cette efficacité ne va pas immédiatement de soi. Plus précisément, l'importance des coordinations s'établit, dans l'économie des conventions, au regard du "fait productif»: une relation est explorée entre qualité des coordinations, qualité des produits et efficacité des ajustements sur le marché des biens.

Il y a là une différence majeure avec la théorie standard étendue. Pour les composantes de celle-ci, l'entreprise se présente comme un lieu de contrats, mais ce fait contractuel est étudié indépendamment du problème de la « qualité » des produits fabriqués dans l'entreprise : l'entreprise apparaît d'abord et avant tout comme un lieu de négociation, à côté de l'autre lieu majeur de négociation qu'est le marché. Les biens produits ont tendance à disparaître derrière la description des arrangements contractuels. L'économie des conventions, à l'inverse, participe de ces courants qui veulent voir avant tout dans l'entreprise un lieu de production, le fait productif n'étant pas intégralement résumé ou « subsumé » par le fait contractuel. Il y a une raison à cette différence. Les différentes problématiques de la TSE se sont construites dans l'objectif de rendre compte de façon positive des phénomènes de prégnance institutionnelle que la TS avait tendance à assimiler immédiatement à des rigidités entravant la flexibilité spontanée du marché. L'économie des conventions, pour sa part, fait faire un basculement à ce questionnement en se demandant si les productions institutionnelles, au lieu de n'être que des palliatifs de l'insuffisance du marché, ne sont pas plutôt des conditions indispensables au fonctionnement d'une économie qui ne juxtapose pas simplement organisations et marchés mais les fait interagir : il n'y a pas seulement des organisations et des marchés manifestant des aptitudes différentes et complémentaires à résoudre les problèmes de coordination, mais des marchés et des organisations qu'il est illusoire de vouloir décrire dans un fonctionnement «pur » ou « idéal », c'est-à-dire indépendamment de la présence de l'autre. Non seulement il n'existe pas de flexibilité pure que les institutions viendraient entraver artificiellement, mais on ne peut pas non plus se satisfaire de l'idée que l'entreprise n'existerait que comme une procédure contractuelle et institutionnelle palliant les défauts du marché. En fait, le succès des organisations, lors des épreuves de vente sur le marché des biens, est moins dépendant de la flexibilité du marché ou des arrangements contractuels internes que de l'efficacité des règles et conventions qui conditionnent la qualité des produits mis à la vente. Implicitement, il y a dans les analyses en termes de conventions une critique de la représentation standard du marché : la concur- 
rence ne se fait pas sur la base de biens homogènes et/ou anonymes, ni même dans un simple contexte d'asymétries informationnelles, mais à travers des produits indissociables de qualités ou caractéristiques intrinsèques qui sont elles-mêmes le reflet des conditions dans lesquelles ils ont été conçus et fabriqués : le produit véhicule irrémédiablement les stigmates (positifs ou négatifs) de ses origines productives et ces stigmates sont déterminants (tout autant ou davantage que les propriétés de flexibilité du marché ou des contrats internes) dans le passage des épreuves de concurrence. Le succès des produits dans ces épreuves est en fait le succès des coordinations qui ont présidé à leur fabrication, étant entendu que ces coordinations ne se réduisent pas à des arrangements contractuels mais importent dans leur forme et leur contenu concrets, dans leur « qualité ». Et il existe à cet égard plusieurs régimes de coordination possibles parce qu'il y a plusieurs types de qualité des coordinations envisageables.

Un aspect de la spécificité de la conception conventionnaliste de l'entreprise apparaît donc à ce niveau : l'entreprise ne s'assimile pas à la réunion contractuelle d'individus mus par leur intérêt ; son analyse doit s'étendre à sa capacité à générer avec ces individus des produits et d'assurer leur vente sur le marché. L'apprentissage collectif et les conventions de qualité et de travail dépassent le fait contractuel et définissent l'entreprise. C'est cette dimension collective ainsi entendue (dépassant la simple combinaison d'intérêts individuels) qui permet de se confronter à l'incertitude des épreuves de marché.

Le collectif, cependant, ne va pas de soi. La coordination n'est pas donnée immédiatement, elle nécessite des règles et des conventions. On a là une différence avec la théorie évolutionniste de la firme. Dans cette théorie, comme chez $O$. Favereau, par exemple, on décèle la même insistance sur l'apprentissage, le même rôle de la connaissance tacite également et les deux courants pourraient revendiquer l'idée, exprimée par O. Favereau que «l'“organisation" n'est plus seulement, comme le marché, un mode d'allocation de ressources dont on se donne l'existence, mais un mode d'allocation de ressources qu'elle crée, en grande partie, elle-même à travers de complexes processus d'apprentissage » (O. Favereau [1989a], p. 298-299 - souligné par O.F.). Mais 1'approche d'O. Favereau, plus que la théorie évolutionniste, suggère que l'apprentissage n'est possible que parce que l'entreprise n'est pas uniquement cet apprentissage mais également un ensemble de règles qui insèrent les salariés dans le collectif d'entreprise, y compris celles (le marché interne) qui les soustraient au marché (externe). Le marché interne n'est pas seulement, comme il peut l'être dans la conception évolutionniste, le cadre qui offre la perspective de stabilité la plus efficace pour la constitution de l'apprentissage ; il est d'abord le mode de coordination qui régit l'insertion des salariés dans l'entreprise et qui leur concède le respect de 1'attente qu'ils expriment de ne plus être soumis au marché. L'entreprise évolutionniste est d'abord un lieu d'apprentissage et secondairement un lieu de coordination, ce que suggère le concept central de « compétences » (skills), lesquelles sont, tout en même temps, celles des « ressources humaines » individuelles et celles de la firme (comme si la coordination des compétences entre elles allait quasiment de soi) ; l'entreprise conventionnaliste est d'abord un lieu de coordination entre des catégories d'acteurs distincts et, sur cette base éventuellement, on peut considérer que se construit un apprentissage. L'optique conventionnaliste est donc de souligner que le collectif n'est pas donné par le seul regroupement, qu'il est une construction de la coordination par les règles. 
Le fait productif intéresse donc l'économie des conventions, au-delà du fait contractuel auquel se limite la TSE et de manière plus large que la théorie évolutionniste. Ce fait productif est cependant particulier si on le compare à celui dont rend compte, par exemple, une théorie comme celle de la régulation, qui semble s'inscrire dans une lignée d'analyse plus traditionnelle en économie. Dans la perspective en termes de conventions, le fait productif s'incarne avant tout dans les règles, les formes, les conventions, bref dans l'instrumentation de la coordination qui, finalement, résume l'entreprise et sa production. Ce n'est pas exactement l'image du fait productif à laquelle se réfère la théorie de la régulation, qui pénère davantage dans la «matérialité » du travail et de la production (la façon dont les salariés entretiennent un certain rapport à la technique, à l'organisation du travail, etc.). Cette théorie s'inscrit dans une tradition issue de Smith et de Marx, celle de l'avance du capital par un capitaliste qui, pour valoriser son capital, doit passer par l'étape consistant à modeler le procès de travail, la technologie, l'organisation. C'est une tradition autre que celle qui a abouti à la TSE et qui, quant à elle, distingue le capitaliste de 1'entrepreneur, simple individu agençant les facteurs de production et réglant des problèmes de coordination. Le traitement du taylorisme est illustratif des différences à ce niveau. L'économie des conventions aborde entièrement le thème du point de vue de l'instrumentation des relations inter-individuelles que permettent les « formes » tayloriennes (cf. le modèle des investissements de forme); cela est mis en correspondance, dans le modèle d'entreprise industriel, avec une certaine qualité des produits. La théorie de la régulation, à la différence, est intéressée par la façon dont le modelage du procès de travail par les principes tayloriens est en mesure de contribuer à façonner les déterminants de productivité. La question est : comment, dans la matérialité de la production et du travail, parvient-on à dégager des gains de productivité ? Le point de vue est différent : il implique que la théorie de la régulation se penchera sur le contenu concret du travail - ce qui justifiera une proximité avec les travaux de sociologie du travail sur le taylorisme et le post-taylorisme; à la différence, l'économie des conventions se tiendra à distance de ces problèmes et s'intéressera davantage à la production et reproduction des règles - ce qui aboutira à se rapprocher des recherches en sciences cognitives, droit du travail, sociologie « de l'accord», etc.

Cette différence n'est pas insignifiante : elle a des implications dans le traitement du rapport entre le micro et le macroéconomique.

\section{« La fin du tout entreprise »?}

Cette caractérisation de la conception conventionnaliste de l'entreprise comme lieu de confrontation collective à l'incertitude étant posée, il reste à risquer une appréciation de son apport. Son intérêt peut certainement être relevé à un double niveau. D'une part, l'économie des conventions, convergente en cela avec la théorie évolutionniste, met en lumière que les mutations qualitatives en cours et les dérèglements économiques qui lui sont liés ne peuvent être appréhendés par une approche trop globalisante et qu'une approche par l'État ou les rapports sociaux qui méconnaîtrait les réalités diverses de l'entreprise est condamnée à un point de vue réducteur. D'autre part, elle montre qu'il n'y a pas de compréhension possible de la firme sans intégration pleine et entière de la dimension du travail, plus précisément en considérant cette intégration comme 
non donnée a priori (par le contrat, les « compétences », etc.). Nous discuterons les limites de ces deux propositions au sein d'une question plus générale : on a pu dire, dans un commentaire sur l'ouvrage de R. Salais et M. Storper [1993], que la perspective des mondes de production représentait « la fin du tout entreprise » (au sens où l'idée d'entreprise se trouve relativisée par la mise en évidence de la diversité qu'elle recouvre) ${ }^{1}$; nous voudrions poser la question de la réalité de cet abandon du « tout entreprise » en lui opposant l'idée que l'économie des conventions pourrait bien avoir «oublié » l'autonomie du social, oubli par lequel elle s'est ainsi sentie autorisée à « rabattre » sur l'entreprise une part importante de l'analyse.

L'économie des conventions met bien en lumière que la coordination ne s'impose pas spontanément par la seule vertu de la rationalité et des contrats, en particulier dans l'entreprise. Mais on a le sentiment que, une fois ce constat fait, les conventions et les règles apparaissent comme des solutions de réintroduction d'une unité ou d'un consensus qui ne sont plus discutés. Il ne s'agit pas de nier l'existence d'intérêts divergents au sein d'une convention : R. Salais ([1991], p. 10) a très justement rappelé que la convention est « une forme qui permet de coordonner des intérêts contradictoires qui relèvent de logiques opposées, mais ont besoin d'être ensemble pour être satisfaits. » De même, il semble également justifié de poser, comme R. Salais, la question de savoir si, «malgré la dissymétrie des rapports de travail qui interdit de les penser selon le modèle du contrat », la relation peut "vraiment fonctionner sans une certaine légitimité de part et d'autre» (ibid., p. 7). Mais cette question doit-elle nécessairement conduire à privilégier une solution (la convention ou la règle) équivalant à l'idée que l'unité l'emporte?

Du point de vue de son unité, l'entreprise conventionnaliste a un statut ambivalent. D'un côté, se présentant comme un lieu de réunion de personnes spécialisées pour faire face collectivement à l'incertitude, son unité n'a, au premier abord, rien d'objectif, cette conception rejoignant, à certains égards, l'interprétation de l'entreprise comme «nœuds de contrats » (R. Salais [1994], p. 380). Mais, d'un autre côté, si les personnes peuvent se trouver ainsi réunies pour une certaine durée, c'est grâce à l'existence d'un cadre commun qui les fait appartenir à un même «monde de production » au regard duquel leurs différences deviennent un fait secondaire pour l'analyse (c'est même le dépassement de leurs divergences d'intérêts initiales qui importe pour le succès du produit). Or, un autre point de vue pourrait aussi bien faire apparaître une ambivalence inverse. Selon celui-ci, l'entreprise constitue bien une unité élémentaire de l'analyse économique : l'unité provient de ce qu'elle est ce que Weber appelait un « compte de capital », c'est-à-dire une unité économique dotée d'une comptabilité faisant apparaître une exigence de rentabilité. Historiquement, cette réalité s'est constituée par l'autonomisation de l' « atelier » à l'égard du foyer de vie familial. Dans cette perspective, l'entreprise se présente comme un lieu dans lequel les actions des intervenants sont conditionnées par et subordonnées aux exigences de reproduction de l'organisation en tant qu'organisation. Ce sont ces exigences qui imposent, pour la cohérence de l'organisation, que les intervenants se réfèrent à un « monde de production » commun. Mais, d'un autre côté,

1. A. Brender, « La fin du "tout entreprise" », Le Monde, 10 mai 1994. 
ces différents intervenants « vivent » leur insertion dans l'entreprise de manière différenciée, parce que, au-delà de leur appartenance à un « monde de production » commun, ils appartiennent à des « mondes sociaux » distincts. L'entreprise s'est dissociée du foyer de vie et les ressorts de l'action de ceux qui la dirigent sont très spécifiques et très limitées, comparées aux logiques de l'action au sein du foyer de vie : ils tiennent - comme Marx l'a montré en opposant la rationalité du capitaliste et le comportement maniaque de l'avare thésaurisant son argent et le soustrayant à la circulation - dans la prise de risque assumée pour les nécessités de reproduction de l'organisation. Les salariés, eux, parce que leur existence ne se concrétise pas dans une comptabilité, demeurent du côté du foyer de vie et donc de logiques d'action qui sont, pour une large part, hétérogènes vis-à-vis des logiques d'action des responsables du compte de capital. Â cet égard, leur insertion dans l'entreprise relève de la nécessité qui, dans une société marchande et monétaire, leur impose de disposer d'un revenu monétaire pour mener leur projet de vie et de ce que, en l'état donné des formations économiques, la viabilité d'une solution indépendante est faible. Cette insertion est donc une « acceptation sous nécessité » (ce qui n'empêche pas, dans un second temps, l'implication dans le travail).

Le salaire traduit cela : il ne peut pas être réduit au prix trouvé dans un accord sur un fait objectif pour les deux parties, qui serait la renonciation à l'indépendance pour bénéficier de la protection collective contre l'incertitude. L'incertitude n'est pas un fait commun aux deux parties : elle est, pour les dirigeants de l'entreprise, le risque assumé comme raison d'être, elle est, pour les salariés, la précarité qu'il faut éviter. Les premiers verront le salaire comme une dépense à consentir pour le développement du « compte de capital » (soit comme un coût qu'il faut s'efforcer de réduire, soit comme une dépense qu'il faut accepter pour obtenir le niveau de qualité, d'effort, d'inventivité requis) ; les seconds considéreront que, étant donné leurs exigences de vie et l'impossibilité de les satisfaire par un autre moyen que l'insertion dans l'entreprise, cette dernière se doit de leur consentir une rétribution leur permettant de réaliser ces exigences. Le contrat de travail n'est pas seulement incomplet, il n'engage pas non plus seulement dans l'établissement de deux principes d'équivalence. Plus fondamentalement, il n'a aucune objectivité commune autre que formelle, car, au plus profond, il correspond à deux engagements différents : le salarié a le sentiment d'échanger la promesse de son travail contre la satisfaction de ses exigences de vie et l'employeur estime, quant à lui, conclure un arrangement sur l'obtention de la réalisation la plus parfaite de ses attentes en échange d'un salaire. Le salaire est donc l'objet d'une incompréhension fondamentale, il n'a pas d'objectivité commune. Il en est de même pour les règles. L'incompréhension est irréductible parce que les intervenants appartiennent à des « mondes sociaux » différents. Le résultat est que l'ambivalence de l'entreprise ainsi redéfinie ne peut trouver de solution en elle-même (comme c'était le cas avec la convention). Il faut un troisième terme, qui est la légitimité, par laquelle les incompréhensions se réduisent. Compréhension et légitimité sont définies par les auteurs conventionnalistes selon une logique instrumentale : ce sont la compréhension du domaine de coopération et la légitimité de la règle d'évaluation grâce auxquelles l'entreprise peut passer les épreuves de réalisation du produit.

Règles, formes, conventions, qui devaient rendre compte de ce que la coordination n'est pas donnée a priori, semblent tourner en objets de consensus. Or ce point de vue est uniquement celui du « compte de capital », sur lequel tout est 
« rabattu ». Le problème de l'économie des conventions, nous semble-t-il, est, en fin de compte, celui de l'inachèvement de son projet consistant à partir du point de vue des acteurs (L. Boltanski, L. Thévenot [1991]) pour élaborer une microéconomie de la compréhension (O. Favereau [1995], p. 515). Si l'on veut adopter pleinement ce point de vue " compréhensif », il faut renoncer à l'hypothèse de rationalité et au "principe d'économie des ressources engagées » (R. Salais, M. Storper [1993], p. 18) pour rejoindre la figure de l'individu empirique, lequel vit dans un monde plus complexe que celui des simples polémiques entre univers de justification. Un premier pas est fait avec la reconnaissance de ce que les marchés internes coïncident avec l'attente des salariés d'être soustraits au marché, mais il faut aller au-delà et reconnaître l'unité contradictoire de l'entreprise, regroupement de mondes sociaux différenciés sous 1'impératif de reproduction de l'organisation.

L'économie des conventions, en suggérant que prévaut, pour l'analyse, le consensus relatif, en vient à nier au social une autonomie qui aurait pu apparaître à partir de la reconnaissance de ce que la coordination n'est pas donnée immédiatement. Sous ce premier point de vue, par conséquent, on ne semble pas avoir affaire, avec l'économie des conventions, à la « fin du tout entreprise »: l' « Entreprise » est remplacée par les «mondes de production », mais c'est bien toujours un point de vue d'offre auquel on a affaire. Cela ne signifie pas que l'entreprise et sa confrontation au marché seraient la seule réalité que donneraient à voir les écrits conventionnalistes. Mais le schéma d'analyse laisse peu de place à des dimensions extra-économiques qui viendraient contraindre l'économique et faire ainsi en sorte que la réalité dépeinte s'avère moins uniforme. Les dimensions non directement économiques sont reconnues par le courant, elles s'incarnent dans les conventions et les divers ordres de justification qui président à la coordination : ainsi en est-il, par exemple, de l'univers domestique. Mais, malgré tout, il serait inexact de voir à partir de cela une réalité structurée par ce que d'autres perspectives appelleraient des « sphères » parmi lesquelles la sphère économique se distingue de la sphère sociale, de la sphère politique, etc. C'est ce schéma en termes de sphères qui est, en revanche, sousjacent à une analyse comme celle du compromis fordiste dans la théorie de la régulation : ce compromis est un compromis entre forces sociales impliquant que des logiques relevant des sphères sociale et politique viennent se confronter aux logiques dominantes de la sphère économique et forcer les comportements économiques à se déplacer vers une solution qui oblige l'économique à reconnaître et à s'accommoder de demandes sociales ou politiques. Le compromis fordiste résulterait ainsi de processus qui dépassent les entreprises et viennent au contraire partiellement les contraindre dans les solutions qu'elles adoptent.

Tel n'est pas le schéma supposé par les analyses en termes de conventions. On peut certes trouver des analyses à première vue assez proches de la logique des sphères, notamment celles qui s'efforcent d'introduire des considérations d'équité à côté des considérations d'efficacité pour réinterpréter l'équilibre macroéconomique (O. Favereau, L. Thévenot [1996]). Mais la notion utilisée de conventions ou de modes de coordination traduit tout autre chose qu'une confrontation entre sphères de la réalité portées par des forces sociales et au terme de laquelle les entreprises pourraient se trouver contraintes par rapport aux souhaits qu'elles auraient développés en dehors de cela. Cela apparaît lorsque le compromis fordiste est relu comme exprimant le fait que « les marchés internes engendrent une croissance suffisante pour rendre supportable leur pro- 
pre poids sans détériorer la situation relative des chômeurs » (ibid., p. 307) (il existe ainsi un taux de croissance pour lequel les règles de gestion des travailleurs s'avèrent compatibles avec l'absence de détérioration de la position des chômeurs sur le marché du travail). C'est là une analyse différente de celle de la théorie de la régulation qui laisse entendre qu'il existe des situations où le social et le politique sont, sous l'effet des compromis entre forces sociales, susceptibles de pousser à la production d'institutions, potentiellement ressenties comme contraignantes par les entreprises mais favorables à la croissance. À la différence, la lecture conventionnaliste gomme tout ce qu'a de problématique et de conflictuel l'existence de règles imposées aux entreprises par des institutions politiques imposant à l'économique des logiques politiques ou sociales.

\section{Quel rapport entre le micro et le macroéconomique?}

Cette différence fait sentir ses effets sur le rôle que l'on fait jouer au microéconomique vis-à-vis de la résolution de difficultés d'ordre macroéconomiques comme, notamment, la persistance du chômage. L'économie des conventions s'est construite sur un effort original pour proposer une interprétation inédite du rapport entre le micro et le macroéconomique et dans l'esprit de dépasser la situation où « les uns n'ont pas la macroéconomie de leur microéconomie » tandis que «les autres n'ont pas la microéconomie de leur macroéconomie » (O. Favereau [1989a], p. 321). Chez O. Favereau, par exemple, l'introduction des organisations équivaut à renouer avec un intérêt pour la croissance macroéconomique : la «soutenabilité » de la croissance est, en quelque sorte, conditionnée par la qualité et l'efficacité du compromis entre, non pas des sphères de la réalité, mais des ordres de justification. La question posée est de savoir comment l'industriel, le marchand, le domestique, etc., peuvent déboucher sur des solutions, s'incarnant dans des règles, qui assurent à la fois l'efficacité microéconomique et le maintien de l'équilibre en contexte de dynamique de croissance, le tout sous considérations d'équité. La convention, les modes de coordination, assurent alors la liaison entre le micro et le macroéconomique. Tel est le sens de l'individualisme méthodologique amendé promu par le courant: le remplacement des forces sociales par des individus situés dans des contextes conventionnels aboutit à la production de règles révélant une dimension collective à la fois issue et autonomisée par rapport aux individus ; ces règles sont à la base de la liaison entre les actions microéconomiques et leurs traductions macroéconomiques.

La question est cependant de savoir ce que deviennent, dans une telle perspective, les «médiations » que, quant au fond, Keynes appelait de ses vœux au terme de son approche macroéconomique. L'approche macroéconomique qu'opposait effectivement cet auteur à l'approche microéconomique néoclassique faisait apparaître que des relations entre catégories agrégées (considérées en masse et en moyenne) avaient la possibilité de faire naître des enchaînements négatifs (récessifs) cumulatifs. Elle concluait à l'impossibilité pour les acteurs individuels de se sortir spontanément de ces enchaînements et donc à la nécessité qu'une médiation les y aide. Cette médiation, pour être efficace, devait échapper aux anticipations individuelles, donc se révéler de manière immédiatement collective, avec ce que cela suppose d'hétérogénéité, voire de contrainte par rapport aux attentes des individus. L'État était cette médiation. 
Que la conscience soit désormais prise de la limite qu'il y a à établir un schéma économique qui sous-estime à la fois les capacités d'anticipation et de réaction des agents économiques face à la politique économique de l'État et leur aptitude à l'innovation et à l'inventivité, cela est une évolution heureuse. Mais la question holiste n'a pas disparu pour autant : n'y a-t-il pas parfois nécessité de voir intervenir des médiations collectives venant éventuellement contrarier les attentes individuelles pour finalement enclencher des mécaniques vertueuses au plan collectif mais aussi individuel ? Simplement, les développements récents de l'analyse économique qui viennent d'être évoqués obligent à se poser cette question en s'interrogeant sur la légitimité de telles médiations et sur la formation de cette légitimité. Cela renvoie à l'affrontement d'argumentaires, qui portent notamment sur les solutions à apporter aux difficultés rencontrées aux plans micro et macroéconomiques (les règles doivent-elles être supprimées et allégées, ou au contraire maintenues, ou renouvelées, mais alors dans quel sens ? etc.). Les argumentaires s'appuient fréquemment sur des logiques de justification. Mais l'insertion des individus dans des «mondes sociaux » différents amène la formation des argumentaires à intégrer des dimensions autres que ces seuls univers de justification, qui ne résument pas l'exhaustivité de l'expérience des individus. C'est cette différence de mondes sociaux dans lesquels se forge l'expérience qui explique la formation d'argumentaires relevant d'une logique ou d'une sphère sociale tandis que d'autres relèvent d'une logique ou d'une sphère économique. Si l'idée de forces sociales dénote un point de vue structuraliste faisant éventuellement trop fi de la liberté individuelle à s'extraire des déterminations sociales, et s'il est justifié de rechercher une articulation plus fine entre l'individuel et le collectif, cependant, il convient sans doute de préserver une capacité à rendre compte de la multi-dimensionnalité de l'expérience individuelle des « mondes sociaux ». L'expérience vécue des mondes sociaux rend crédible la définition de catégories sociales et économiques agrégées fondant, sur le plan analytique, la possibilité d'une approche macroéconomique au sens strict de l'expression (c'est-à-dire une approche holiste) et justifiant pour cette analyse de rechercher des compromis sociaux entre ces catégories, des compromis susceptibles de fonder un régime de croissance durable. Simplement, il reste à développer la connaissance de l'alchimie qui transforme les expériences individuelles en ces catégories d'individus pouvant être agrégés. En ce sens, l'apport de l'économie des conventions doit être appréhendé, non plus seulement du point de vue de ce qu'elle offre de directement utilisable dans l'analyse, mais cette fois dans le rôle d' " aiguillon » de la réflexion qu'elle est susceptible de constituer pour un renouvellement d'une approche holiste dont on n'a fait ici que suggérer la possibilité et l'intérêt.

\section{CONCLUSION}

Depuis près de vingt ans, l'économie des conventions développe une approche spécifique au sein de laquelle il est possible de dégager les éléments d'une conception particulière de l'entreprise. Trop souvent assimilée par des lectures insuffisamment attentives, soit à une variante française des théories des contrats, soit à une variante individualiste de la théorie de la régulation, l'économie des conventions fournit des résultats qui, précisément, interrogent (parmi d'autres) 
ces deux ensembles d'analyses. En rupture avec la TSE qui tend à ne plus voir dans l'entreprise que des contrats inter-individuels, l'économie des conventions réhabilite la dimension productive en même temps qu'elle remet en cause la représentation standard du marché et de la concurrence : le succès des entreprises dans la concurrence repose avant tout sur ses produits, lesquels véhiculent la qualité des coordinations internes. Par là, l'analyse ne renvoie pas tant aux contrats qu'aux conventions et à l'apprentissage collectif qui conditionnent les caractéristiques concrètes des marchandises. Le produit est ce qui singularise l'entreprise dans la concurrence. Il y a donc également là un déplacement certain vis-à-vis d'une approche comme la théorie de la régulation, qui tire de son héritage marxien la focalisation sur un acteur « symbolique » incarnant la catégorie économique des entreprises (le capitaliste chez Marx, la firme fordiste dans la théorie de la régulation) par-delà la singularité de chacun. Cela conduit à deux représentations très différentes de la liaison entre le micro et le macroéconomique. L'économie des conventions propose un schéma d'articulation original, qui interroge à la fois la TSE dans sa capacité à renouer, à partir de la perspective contractuelle, avec des énoncés macroéconomiques et, inversement, les difficultés d'une approche holiste comme celle de la régulation à se donner une microéconomie pertinente. La présentation des thèses conventionnalistes a permis ici d'expliciter cet apport au sein de l'analyse économique contemporaine prise dans sa diversité. Mais leur discussion critique s'est employée à montrer que la proposition conventionnaliste d'un individualisme méthodologique renouvelé laisse sans réponses un certain nombre de questions posées par la perspective holiste et qu'elle laisse donc ouverte la voie de l'engagement d'un programme de travail destiné à enrichir le point de vue holiste sur la base d'une représentation légèrement différente de l'individu et de la société.

\section{RÉFÉRENCES BIBLIOGRAPHIQUES}

AMBLARD H., BERnOuX Ph., HeRreros G., LiviAN Y.-F. [1996], Les nouvelles approches sociologiques des organisations, Paris, Le Seuil.

BoISARD P., LETABLIER M.-T. [1987], «Le camembert : normand ou normé. Deux modèles de production dans l'industrie française », Cahiers du Centre d'études de l'emploi, $\mathrm{n}^{\circ} 30$, « Entreprises et produits ».

Boltanski L., THÉVENOT L. [1991], De la justification. Les économies de la grandeur, Paris, Gallimard.

EYMARD-DUVERNAY F. [1986], « La qualification des produits », dans SALAIS R., THÉVENOT L. [1986].

EYMARD-DUVERNAY F. [1987], «Les entreprises et leurs modèles », introduction à : Cahiers du Centre d'études de l'emploi, $\mathrm{n}^{\circ} 30$, « Entreprises et produits ».

EYMARD-DUVERNAY F. [1989], "Conventions de qualité et formes de coordination », Revue économique, 40 (2).

EYMARD-DUVERNAY F. [1994], « Coordination des échanges par l'entreprise et qualité des biens », dans ORLÉAN A. [1994]. 
FAVEREAU O. [1986], « La formalisation du rôle des conventions dans l'allocation des ressources », dans SALAIS R., THÉVENOT L. [1986].

FAVEREAU O. [1988], «La "Théorie générale" : de l'économie conventionnelle à l'économie des conventions », Cahiers d'économie politique (14-15).

FAVEREAU O. [1989a], « Marchés internes, marchés externes », Revue économique, 40 (2).

FAVEREAU O. [1989b], « Organisation et marché », Revue française d'économie, IV (1).

FAVEREAU O. [1989c], « Vers un calcul économique organisationnel », Revue d'économie politique, 99 (2).

FAVEREAU O. [1995], « Conventions et régulation », dans BOYER R., SAIllard Y., Théorie de la régulation. L'état des savoirs, Paris, La Découverte.

FAVEREAU O., THÉVENOT L. [1996], «Réflexions sur une notion d'équilibre utilisable dans une économie de marchés et d'organisations », dans BALlOT G. (dir.), Les marchés internes du travail : de la microéconomie à la macroéconomie, Paris, PUF.

OrléAN A. (dir.) [1994], Analyse économique des conventions, Paris, PUF.

RAMAUX C. [1996], «Les asymétries et les conflits sont-ils solubles dans la cognition? Une lecture critique des Économies de la grandeur de L. Boltanski et L. Thévenot (1991) ", Économies et sociétés, série D n² 2, 30 (9).

REYNAUD B. [1988], « Le contrat de travail dans le paradigme standard », Revue française d'économie, 3 (4).

REYNAUD B. [1992], Le salaire, la règle et le marché, Paris, Christian Bourgois.

REYNAUD B. [1994], Les théories du salaire, Paris, La Découverte, coll. « Repères ».

SALAIS R. [1989], «L'analyse économique des conventions du travail », Revue économique, 40 (2).

SALAIS R. [1991], «Flexibilité et conventions du travail : une approche », Économie appliquée, XLIV (2).

SALAIS R. [1994], « Incertitude et interactions de travail : des produits aux conventions », dans ORLÉAN A. [1994].

SALAIS R., STORPER M. [1993], Les mondes de production. Enquête sur l'identité économique de la France, Paris, Éditions de l'École des hautes études en sciences sociales.

SAlAis R., THÉVENOT L. (dir.) [1986], Le travail. Marchés, règles, conventions, Paris, Economica.

THÉVENOT L. [1985], "Les investissements de forme », Cahiers du Centre d'études de l'emploi, «Conventions économiques ».

THÉVENOT L. [1989], « Équilibre et rationalité dans un univers complexe », Revue économique, 40 (2). 